\title{
Plasticity in the Human Speech Motor System Drives Changes in Speech Perception
}

\author{
DDaniel R. Lametti, ${ }^{1,2}$ Amélie Rochet-Capellan, ${ }^{3}$ Emily Neufeld, ${ }^{1}$ Douglas M. Shiller, ${ }^{4,5,6}$ and David J. Ostry ${ }^{1,7}$ \\ ${ }^{1}$ Department of Psychology, McGill University, Montréal, Quebec, Canada H3A 1B1, ${ }^{2}$ Institute of Neurology, University College London, London, United \\ Kingdom, WC1N 3BG, ${ }^{3}$ GIPSA-LAB, Département Parole and Cognition, CNRS and Grenoble Université, Grenoble, France, ${ }^{4}$ School of Speech Pathology and \\ Audiology, Université de Montréal, Montréal, Quebec, Canada H3N 1X7, ${ }^{5}$ Research Center, Sainte-Justine Hospital, Université de Montréal, Montréal, \\ Quebec, Canada H3T 1C5, ${ }^{6}$ Centre for Research on Brain, Language \& Music, Montréal, Canada H3G 2A8, and ${ }^{7}$ Haskins Laboratories, New Haven, \\ Connecticut 06511
}

Recent studies of human speech motor learning suggest that learning is accompanied by changes in auditory perception. But what drives the perceptual change? Is it a consequence of changes in the motor system? Or is it a result of sensory inflow during learning? Here, subjects participated in a speech motor-learning task involving adaptation to altered auditory feedback and they were subsequently tested for perceptual change. In two separate experiments, involving two different auditory perceptual continua, we show that changes in the speech motor system that accompany learning drive changes in auditory speech perception. Specifically, we obtained changes in speech perception when adaptation to altered auditory feedback led to speech production that fell into the phonetic range of the speech perceptual tests. However, a similar change in perception was not observed when the auditory feedback that subjects' received during learning fell into the phonetic range of the perceptual tests. This indicates that the central motor outflow associated with vocal sensorimotor adaptation drives changes to the perceptual classification of speech sounds.

Key words: action; motor learning; perception; plasticity; speech

\section{Introduction}

There has been recent interest in the idea that the effects of motor learning are associated with changes to perceptual function and sensory networks in the brain. Evidence in support of this idea has been reported for limb movement and speech, where in both cases changes in perception are observed to accompany adaptation (Cressman and Henriques, 2009; Nasir and Ostry, 2009; Shiller et al., 2009, 2010; Vahdat et al., 2011, 2014). However, the source of the perceptual effects is unclear. In speech, motor learning involves changes to both the motor commands that drive the articulators and the auditory and somatosensory signals received during training. So what produces the perceptual change during motor learning? Is it the change to motor function that occurs during learning? Is it perceptual learning related to the altered sensory inputs? Or is it some combination of the two?

A number of recent studies have explored perceptual change in the context of human arm movement (Haith et al., 2008;

\footnotetext{
Received Jan. 9, 2014; revised June 16, 2014; accepted June 20, 2014.

Author contributions: D.R.L., A.R.-C., D.M.S., and D.J.O. designed research; D.R.L. and E.N. performed research; D.R.L. analyzed data; D.R.L., A.R.-C., D.M.S., and D.J.O. wrote the paper.

This work was supported by the National Institute on Deafness and Other Communication Disorders Grant DC012502, the Natural Sciences and Engineering Research Council of Canada (NSERC), and Les Fonds Québécois de la Recherche sur la Nature et les Technologies, Québec (FQRNT). D.R.L. was supported by a postdoctoral fellowship from FQRNT. E.N. was supported by an Undergraduate Student Research Award from NSERC.

The authors declare no competing financial interests.

Correspondence should be addressed to David J. Ostry, Department of Psychology, McGill University, Montréal, Quebec, Canada H3A 1B1. E-mail: david.ostry@mcgill.ca.

DOI:10.1523/JNEUROSCI.0108-14.2014

Copyright $\odot 2014$ the authors $\quad 0270-6474 / 14 / 3410339-08 \$ 15.00 / 0$
}

Cressman and Henriques, 2009; Ostry et al., 2010; Vahdat et al., 2011, 2014). Perceptual change has also been reported in studies of speech motor learning in response to auditory (Shiller et al., 2009) and somatosensory perturbations (Nasir and Ostry, 2009). In work on limb movement, changes in sensed limb position occur in a direction associated with the central motor outflow needed to compensate for the mechanical load (or altered visual input).

Here we altered in real time the auditory feedback participants received as they produced speech (Houde and Jordan, 1998; Purcell and Munhall, 2006; Villacorta et al., 2007; Cai et al., 2010; Rochet-Capellan and Ostry, 2011; Lametti et al., 2012, 2014). We examined both the motor adaptation that occurs to correct for the perturbation and associated changes in the perception of speech sounds. As a speech-learning task, subjects repeated the word "head." We either shifted the frequency of the first formant (F1) downward during the production of head, to make it sound more like "hid," or we shifted it upward toward "had." Following learning, we examined changes in the classification of speech sounds. To distinguish whether the observed perceptual change was due to what the subjects heard versus what subjects said during adaptation, we synthesized two perceptual continua, one between head and had, and the other between head and hid. Consequently, depending on the direction of the feedback perturbation, subjects received auditory feedback during adaptation that was within one of these continua and generated vocal motor outputs that corresponded to sounds in the other. Our finding was that the perceptual change that accompanied motor learning was associated with the utterances produced to achieve compen- 
sation rather than with the acoustical input that the subject received during adaptation. Changes in perception were greatest when acoustical changes in speech production that accompanied learning fell into the phonetic range of the perceptual tests. This is consistent with the idea that changes to central motor commands associated with speech learning are the source of changes observed in the perceptual classification of speech sounds.

\section{Materials and Methods}

Subjects, apparatus, task. Ninety-eight subjects ( 76 females) between the ages of 18 and 30 participated in the experiments. Eighty subjects were recruited for the main study; 18 additional subjects participated in a control study. The McGill University Faculty of Medicine Institutional Review Board approved the experimental protocol. All subjects gave their informed consent before participating and reported being native English speakers with normal hearing and speech. Testing was done in a sound-attenuating chamber. Subjects wore headphones (Stax SR001MK2) and sat comfortably at a desk in front of a computer. Subjects produced words as they appeared on the computer screen and a directional microphone (Sennheiser) recorded speech. As subjects spoke, they heard the sound of their own voice from headphones mixed with $70 \mathrm{~dB}$ speech-shaped masking noise. Subjects also made perceptual judgments about words played from the headphones by pressing keys on the computer's keyboard.

Procedure. The experiment began with a baseline production phase consisting of 90 utterances of unaltered speech. Subjects then performed the first perceptual test. This baseline perceptual test was followed by 180 utterances in which the sound of the voice was altered in real time to induce speech motor learning (see below, Speech motor learning). A second perceptual test followed speech motor learning, after which subjects produced another 180 utterances with unaltered speech to "washout" the effects of motor learning. This phase of the experiment included a 5 min break in which subjects could leave the testing area. A final perceptual test followed washout production.

Subjects were randomly divided into two groups based on the speech perceptual test they performed. In Experiment 1, 41 subjects always made perceptual judgments about a 10-step continuum of words that spanned the distinction between head and had (see below, Measuring speech perception). These subjects produced head and had an equal number of times in a random order during baseline production and washout, and only produced head during the motor learning phase of the experiment. In Experiment 2, 39 subjects always made perceptual judgments about a 10-step continuum of words that spanned the perceptual distinction between head and hid. These subjects produced head and hid an equal number of times in a random order during baseline production and washout, and only produced head during the motor learning phase of the experiment. Within each experiment, subjects were subdivided into three groups: two speech motor-learning groups that differed in how their voice was altered and a control group that did not have their voice altered. Subjects in the control groups only performed the first two perceptual tests, and only produced 90 utterances during the washout phase of the experiment.

Measuring speech perception. In Experiment 1, perception was measured using a continuum of words that spanned the perceptual distinction between head and had. The continuum was based on words provided by a male Canadian English speaker. Ten experimental stimuli were created in which the first two formants in the word head were shifted in equal steps toward formant values in had. F1 and F2 for head were 519.25 and $1738.30 \mathrm{~Hz}$, respectively, and F1 and F2 for had were 716.62 and $1563.04 \mathrm{~Hz}$, respectively. During each perceptual test, subjects heard each of the 10 stimuli 21 times in a random order; after each stimulus they were prompted by instructions on the computer screen to press one key if they thought the stimulus sounded more like head or to press another key if they thought it sounded more like had. Subjects pressed the spacebar to trigger the next stimulus. Perception was measured in a similar manner during Experiment 2, but the perceptual stimuli spanned the distinction between head and hid. This 10-step continuum was created based on the pure head stimulus from Experi- ment 1 and an instance of hid taken from the same male speaker. F1 and F2 for hid were 434.11 and $1787.20 \mathrm{~Hz}$, respectively.

For each perceptual test, the probability that a subject would report hearing head for a particular stimulus was computed. Psychometric functions were fit to these probabilities using the binomial distribution fitting method (glmfit in MATLAB). From the psychometric function the perceptual boundary - the point on the continua where head was perceived $50 \%$ of the time-was computed. Learning-related changes in speech perception were assessed by comparing the perceptual boundary before speech motor learning to the perceptual boundary after speech motor learning. Within each experiment, changes in perception were compared between groups using one-way ANOVAs. The significance level was 0.05 , and it was corrected for multiple post hoc comparisons (in this case, unpaired two-tailed $t$ tests) using the Holm-Bonferroni method.

Speech motor learning. As an experimental model of speech motor learning, the first formant frequency (F1) of the vowel sound in head was altered in real time using an acoustical effects processor (VoiceOne; TCHelicon) and filters (Fig. 1A). The effects processor altered $\mathrm{F} 1$ in proportion to baseline $\mathrm{F} 1$ frequency, shifting $\mathrm{F} 1$ down by $\sim 18 \%$ or up by $\sim 24 \%$. In Experiment 1, during the motor learning phase of the experiment, one group of subjects $(N=13)$ had their F1 shifted up by $175.74 \mathrm{~Hz}$ (SD $25.62)$, toward the $\mathrm{F} 1$ in had and a second group of subjects $(N=13)$ had their F1 shifted down by $-139.05 \mathrm{~Hz}$ (SD 31.21), toward the F1 in hid; a third group of subjects $(N=12)$ simply produced the word head without altered feedback. Similarly, in Experiment 2, one group of subjects $(N=$ 15) had their F1 shifted up by $177.32 \mathrm{~Hz}$ (SD 41.43), and a second group $(N=13)$ had their F1 shifted down by $-127.56 \mathrm{~Hz}$ (SD 26.42); a third group of subjects $(N=10)$ simply produced the word head without altered feedback. Four subjects were excluded - three from Experiment 1 and one from Experiment 2-as they failed to produce significant changes in F1 frequency in response to altered auditory feedback.

Auditory analysis. Speech was recorded at $44.1 \mathrm{kHz}$. The software package Praat detected vowel boundaries and calculated the first formant frequency for each utterance based on a $30 \mathrm{~ms}$ window at the center of the vowel (Rochet-Capellan and Ostry, 2011; Lametti et al., 2012, 2014). To examine changes in F1 related to speech motor learning, the data were normalized to remove between-subject differences in baseline F1 frequency. To do this, on a per subject basis, the mean F1 of the baseline utterances was subtracted from the F1 of each utterance. To assess whether individual subjects learned to compensate for changes in auditory feedback, the mean of the last 45 altered-feedback utterances was compared with the mean of baseline F1 using one-tailed $t$ tests. The mean F1 relative to baseline of the last 45 altered-feedback utterances was compared across groups using one-way ANOVAs. The significance level for these tests was 0.05 , and it was corrected for multiple post hoc comparisons as above using the Holm-Bonferroni method.

Listening control study. To assess the possibility that the auditory input that accompanies learning might on its own change the perception of speech sounds, we examined the effect on speech perception of listening to speech motor learning. Eighteen new subjects were recruited for this test. The experiment began with a baseline production phase consisting of 45 utterances of head and 45 utterances of hid produced in a random order. Subjects then performed the head-to-hid perceptual test described previously before listening to recordings of speech motor learning. The recordings were drawn at random from the 15 participants in Experiment 2 who had the $\mathrm{F} 1$ of their head productions shifted up in frequency. We used this material as stimuli because this group showed the greatest perceptual change. The recordings were taken from the speech motor learning phase of the experiment and they included the speech-shaped masking noise heard throughout speech motor learning. A second headto-hid perceptual test followed listening to speech motor learning, after which subjects again produced 45 utterances of head and 45 utterances of hid in a random order.

\section{Results}

We assessed whether the perceptual changes that occur in conjunction with speech motor learning arise as a result of the auditory feedback that subjects receive or whether they are related to 
A

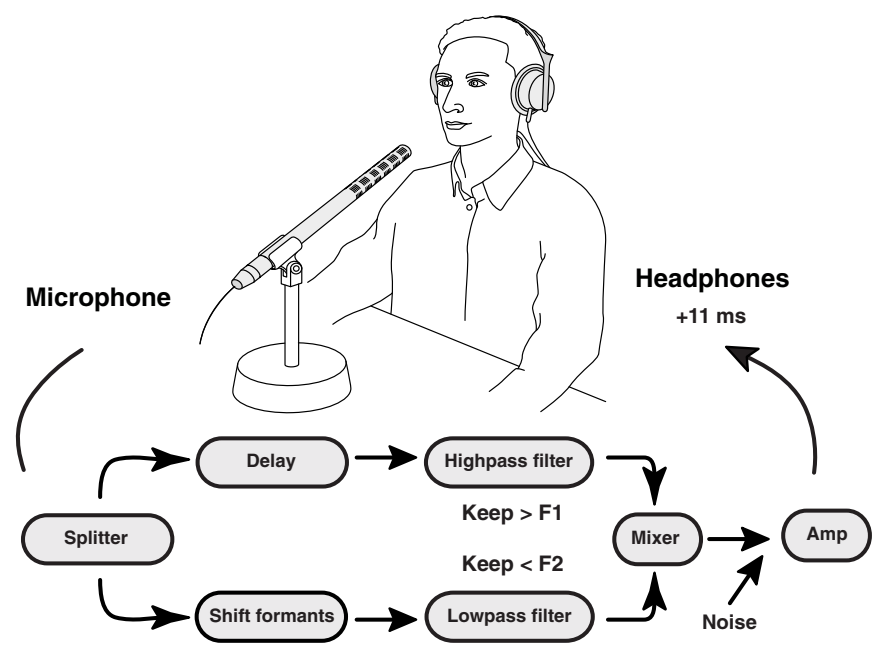

B
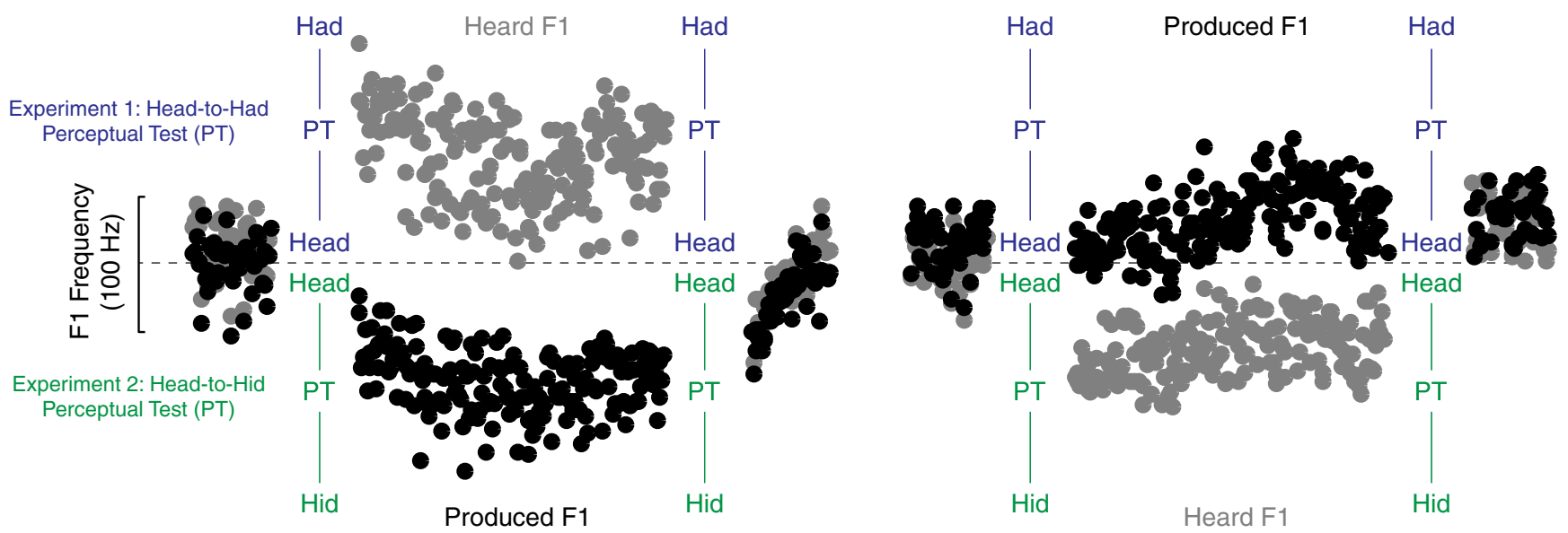

Figure 1. Experimental setup and hypothesis. A, Participants produced the word head into a microphone. An acoustical effects processor and audio filters were used to alter the first formant frequency (F1) of the vowel sound in head. This altered speech sound was played back to subjects through headphones with an 11 ms delay. Participants produced head but heard themselves through the headphones producing something that sounded more like had or hid, depending on the direction of the F1 alteration. Changes in F1 production in compensation for the F1 alteration were used as a measure of sensorimotor adaptation. $\boldsymbol{B}, \mathrm{In}$ Experiment 1 the perceptual boundary between the words head and had was measured before and after participants learned to compensate for altered auditory feedback. In one group, altered feedback drove the perception of learning (i.e., what subjects "heard") into the phonetic range of the head-to-had perceptual test (PT); in a second group, altered feedback drove learned changes in production into the phonetic range of the head-to-had perceptual test. Perceptual change on the head-to-had continuum was only predicted when changes in speech movements fell into the range of the perceptual test. In Experiment 2 the perceptual boundary between the words head and hid was measured before and after participants learned to compensate for altered auditory feedback. Again, two groups of subjects were tested: in one case the perception of learning fell into the phonetic range of the perceptual test; in the other case learned changes in production fell into the phonetic range of the perceptual test. Perceptual change on the head-to-hid continuum was predicted when learned changes in speech movements fell into the range of the perceptual test.

changes in motor function that occur in relation to learning. In Experiment 1 we measured how subjects categorized the words head and had by evaluating the perceptual boundary between the two words before and after subjects performed a speech motor learning task that changed the way they produced head. Speech motor learning was induced by increasing or decreasing in real time the first formant frequency (F1) of the vowel sound in head so that subjects perceived themselves producing a word with an F1 closer to that in had or hid (Fig. 1A). The perceptual boundary between head and had was measured using a two-alternative forced choice perceptual test consisting of 10 stimuli that spanned the vowel-sound contrast between head and had. Our aim here was to determine whether perceptual change would be associated with those cases in which subjects' auditory input or, alternatively, their vocal output, fell into the phonetic range of the perceptual test (Fig. 1B).
In Experiment 1, subjects were divided into three groups. After a period of baseline production followed by a baseline measurement of speech perception, the "up-shift" group (red data) had the $\mathrm{F} 1$ of their head productions increased by $+175.74 \mathrm{~Hz}$ on average so that they perceived themselves producing an utterance that was closer to had. The "down-shift" group (blue data) had their F1 decreased by $-139.05 \mathrm{~Hz}$ on average so that they perceived themselves producing an utterance closer to hid. The "noshift" group simply produced head without having their voice altered. Figure $2 A$ shows the speech motor learning data for Experiment 1 . Change in the produced F1 relative to baseline for each utterance is plotted over the course of the experiment. The up-shift group responded to an F1 increase by learning to produce F1 at a lower frequency (red data). The down-shift group responded to an $\mathrm{F} 1$ decrease by learning to produce $\mathrm{F} 1$ at a higher frequency (blue data). The no-shift group showed a small de- 
crease in F1 (black data). Following altered feedback, F1 values returned to baseline levels after a period of washout production.

Figure $2 B$ shows the amount of $\mathrm{F} 1$ change for the last 45 trials produced with altered auditory feedback. The solid bars represent the F1 that subjects produced and the outlined bars represent the F1 that subjects heard; that is, the altered F1 that came out of the headphones. Motor learning-related changes in $\mathrm{F} 1$ production were significantly different $(p<0.01)$ from changes in production associated with simply producing unaltered speech (one-way ANOVA: $F_{(2,35)}=38.44, p<$ 0.001). For the up-shift group (red), speech motor learning resulted in a $-59.67 \mathrm{~Hz}$ change in the produced $\mathrm{F} 1$ value. For the down-shift group (blue), learning resulted in a $+50.31 \mathrm{~Hz}$ change in F1. The no-shift group (black), which produced unaltered speech and did not experience motor learning, showed a $-16.35 \mathrm{~Hz}$ change in F1. Altered auditory feedback thus placed subjects' acoustical inputs in the phonetic range of the words head and had for the up-shift group (red), while driving learned changes in speech production into this phonetic range for the down-shift group (blue).

Did speech motor learning alter speech perception? Figure 3, $A-C$, shows psychometric functions fit to the mean proportion of had responses for each stimulus (colored circles) before and after speech motor learning, or, in the case of the no-shift group, repeated production. Changes in the perceptual boundary are quantified in the left side of Figure $3 D$. Perceptual change following speech motor learning was compared between groups (oneway ANOVA: $\left.F_{(2,34)}=3.849, p=0.031\right)$. Only the down-shift group showed a significant change in perception compared with the up-shift group and the no-shift group $(p=0.044, p=0.011$, respectively). Following speech motor learning, subjects in the down-shift group became more likely to report hearing had. Changes in speech perception in the up-shift group and the noshift group were not different from each other or zero $(p=0.93)$. The right side of Figure $3 D$ shows that the perceptual change in the down-shift group returned to zero after learning was washed out. This suggests that the perceptual change depended on the maintenance of the adapted state.

In the case of the down-shift group in Experiment 1, altered auditory feedback led to adaptation-related changes in speech production that shifted vocal output into the phonetic range of the head-to-had perceptual test and a change in speech perception was observed. However, in the case of the up-shift group, altered feedback placed auditory inputs in the phonetic range of the perceptual test and changes in perception were not observed. This suggests that perceptual change during motor learning is driven by changes in the motor system. To further explore the idea, we repeated Experiment 1 with new subjects and a new 10 -step perceptual continuum that spanned the vowel sound contrast between head and hid. Using this new continuum we reasoned that adaptation-related changes in speech production in response to increases in F1 would now fall into the phonetic
B

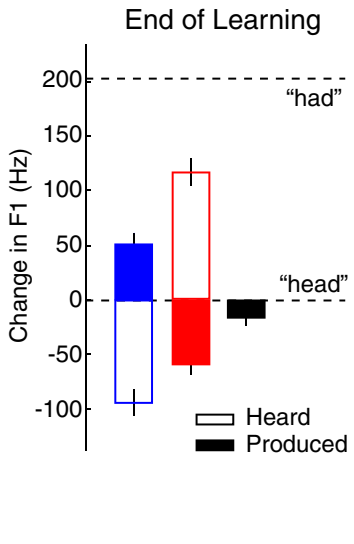


A

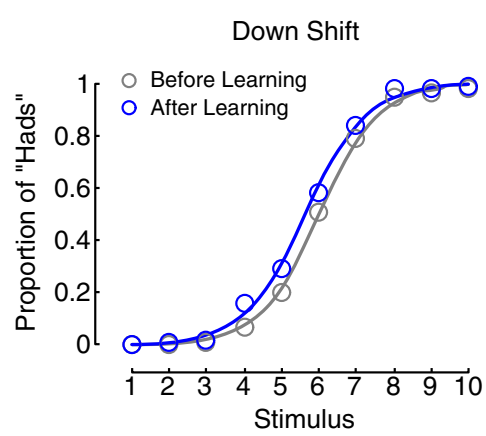

C

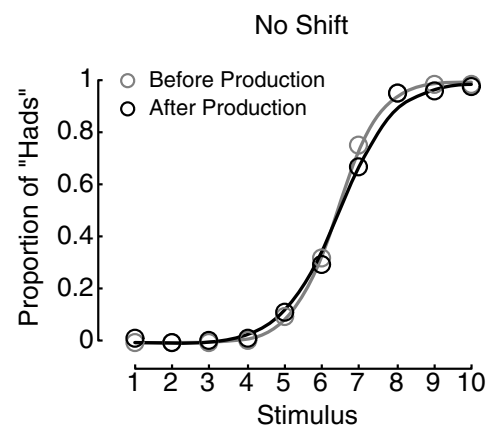

B

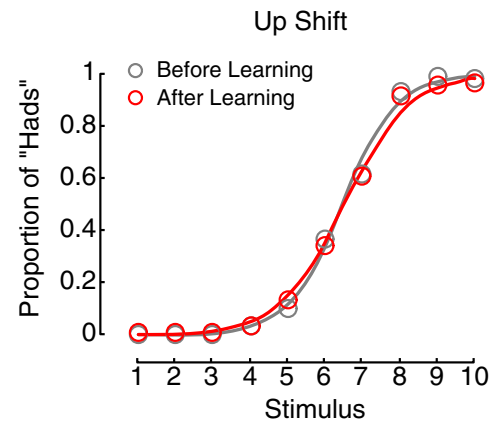

D

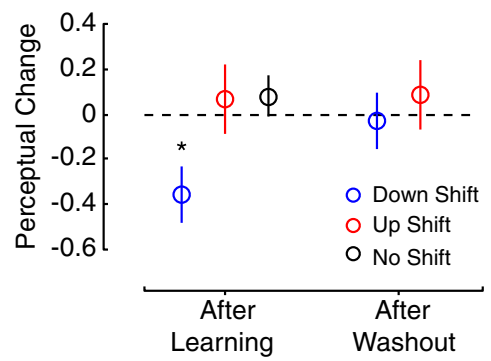

Figure 3. Speech motor learning altered speech perception. $\boldsymbol{A}-\boldsymbol{C}$, Show psychometric functions fit to the average proportion of had responses (represented by the unfilled circles) at each stimulus before (gray lines) and after (colored lines) speech motor learning. $\boldsymbol{D}$, After speech motor learning, a significant change in the perceptual boundary was only observed in the case of the down-shift group (blue circle). This perceptual change disappeared after washout trials that eliminated motor learning; ${ }^{*} p<0.05$.

A

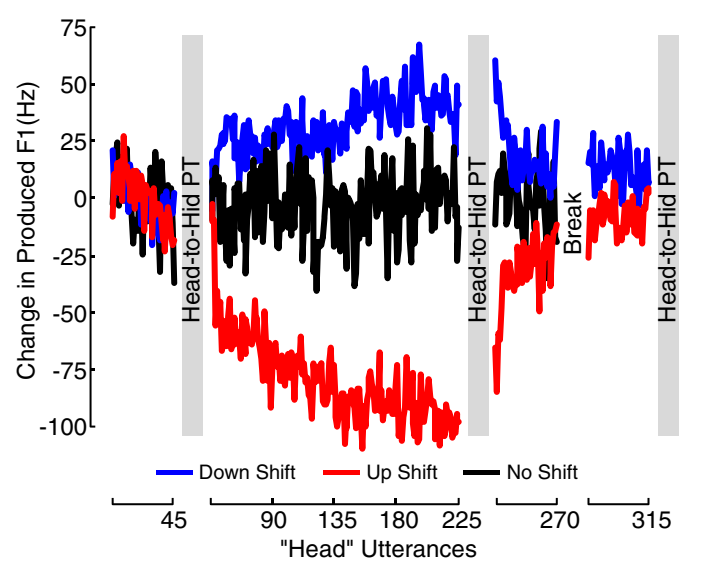

B

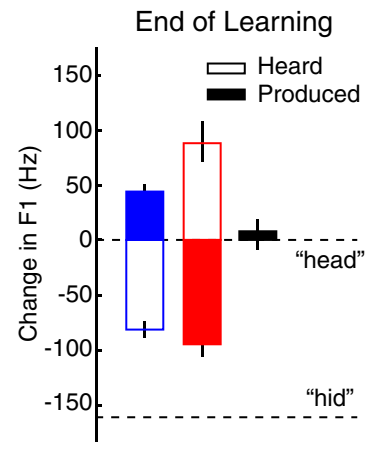

Figure 4. Experiment 2: altered auditory feedback drove learned changes in speech production. $\boldsymbol{A}$, Using new participants, Experiment 1 was repeated with head-to-hid perceptual tests. After baseline production, feedback of F1 was either increased (red, up-shift group), decreased (blue, down-shift group), or left unaltered (black, no-shift group). A period of washout production followed altered auditory feedback. The solid lines join the mean F1 for each head utterance. Mean produced F1 is plotted relative to baseline F1 production. Participants performed head-to-hid perceptual tests before altered feedback, after altered feedback, and after washout trials (location of gray bars). $\boldsymbol{B}$, The solid bars represent the mean produced F1 from head over the last 45 utterances of altered feedback compared with baseline F1 from head; the unfilled bars represent the mean F1 that came out of the headphones over the last 45 utterances of altered feedback compared with baseline F1. The dashed line represents the average F1 taken from baseline utterances of the word hid compared with baseline F1 production of head. The down-shift group (blue) learned to produce F1 at a higher frequency during altered feedback, the up-shift group (red) learned to produce F1 at a lower frequency during altered feedback, and the no-shift group (black) showed no change in F1 production. Error bars represent $\pm 1 \mathrm{SE}$.

change following speech motor learning was compared between groups (one-way ANOVA: $F_{(2,35)}=6.82, p<0.01$ ). In this case, the up-shift group - the only group whose altered production fell into the phonetic range of the perceptual test—-showed a signifi- cant change in perception compared with the down-shift group $(p=0.0059)$ and the no-shift group ( $p=0.0033)$. Following speech motor learning, subjects in the up-shift group became more likely to report hearing hid. The right side of Figure $5 D$ shows that this perceptual change was significantly reduced after speech motor learning was washed out. For the downshift group, whose auditory inputs during learning fell into the range of the perceptual test, changes in speech perception were not different from changes associated with the repeated production of unaltered speech $(p=0.42)$. In combination with Experiment 1, the results show that alterations in speech perception that accompany speech motor learning are associated with changes in motor function due to learning, and not with altered auditory input.

Figure 6 shows the results of a control study run to examine whether changes in speech perception occur simply by listening to speech motor learning. The blue, red, and black points at the left represent changes in perception following motor learning or repeated production for the down-shift, up-shift, and no-shift groups (respectively) from Experiment 2. The green point at the right represents data from a new a group of subjects; the point depicts changes in the perceptual boundary between head and hid following listening to subjects in the Experiment 2 up-shift group as they learned to compensate for altered auditory feedback. Perceptual change following speech motor learning, repeated production, and listening to speech motor learning was compared between groups (one-way ANOVA: $\left.F_{(3,53)}=4.0, p=0.012\right)$. The up-shift group showed more perceptual change than the other three groups. Learning to produce speech within the phonetic range of the perceptual test resulted in a greater perceptual change than simply listening to the same behavior $(p=0.015)$. This provides further evidence that speech motor learning-related changes in the motor system drive changes in speech perception.

We compared the amount of learning between the two experiments. We found that the down-shift groups showed no difference in the amount of learning $\left(t_{(29)}=\right.$ $0.58, p=0.56)$. The up-shift group in Experiment 2 showed more learning than the up-shift group in Experiment $1 t_{(24)}$ $=2.11, p=0.045)$ even though the size of the F1 alteration was similar between these two groups. This presumably relates to large individual differences in the amount of compensation for altered auditory feedback (Lametti et al., 2012). 
Finally, we tested the relationship between compensation for altered auditory feedback and perceptual change for the two motor-learning groups that showed a significant change in perception compared with their respective no-shift control groups. The four subjects that were originally excluded because they failed to compensate for altered auditory feedback (see Materials and Methods) were included in this analysis. In the case of the down-shift group from Experiment 1, the correlation between the amount of speech motor learning and changes in the perceptual boundary between head and had was $-0.06(p=0.81)$. In the case of the upshift group from Experiment 2, the correlation between the amount of speech motor learning and changes in the perceptual boundary between head and hid was $0.39(p=0.16)$. Thus, the amount of speech motor learning did not predict the amount of perceptual change.

\section{Discussion}

Motor learning involves changes to both sensory and motor functions of the brain. It requires the establishment and shaping of sensory targets and the development of motor commands that achieve these perceptual goals (Darainy et al., 2013; Vahdat et al., 2014). Changes to both motor and sensory systems with learning can be seen here as systematic changes to the perceptual classification of speech sounds in conjunction with speech motor learning. The present scientific challenge is that perceptual and motor learning occur in parallel and as a result, for both motor and perceptual function, it is difficult to know whether the changes that are seen are due to perceptual systems or to refinements of movement in the context of learning.

In the present set of studies we have been able to separate sensory from motor factors that contribute to the perceptual changes in speech motor learning (Houde, 2009). We have done this by using perceptual tests that were directly related to auditory inputs that subjects received during learning versus the vocal motor outputs that occur in conjunction with adaptation. In this way we could determine whether perceptual changes that we observed were related to what subjects heard during learning as opposed to what they produced. Our experimental manipulation involved adaptation to altered auditory feedback during speech production. We observe that perceptual changes associated with motor learning are related to the utterances that subjects produced to achieve compensation rather than to the altered acoustical input that provides the sensory stimulus for learning. Furthermore, the perceptual change is only observed when subjects are in the adapted state. The perceptual change is thus a result of changes in motor function.

The perceptual changes that we observed result in a reduction or narrowing of the range of perceptually acceptable productions of the training utterance. This is true for both of the perceptual continua that we tested. That is, for both the head-hid continuum and the head-had continuum, adaptation to altered auditory feedback during repetition of the utterance head, resulted in
Down Shift

Learning

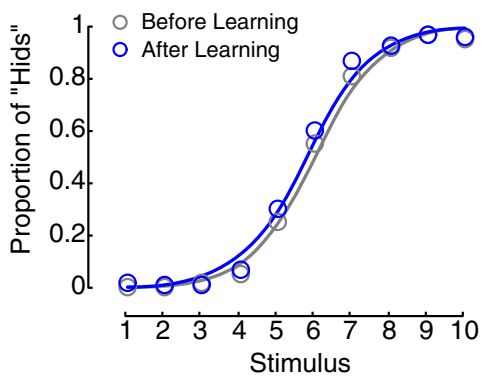

No Shift

Production

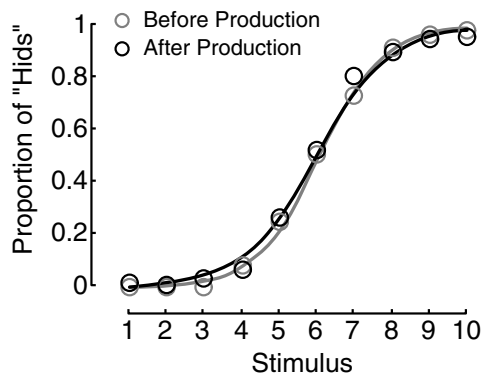

B

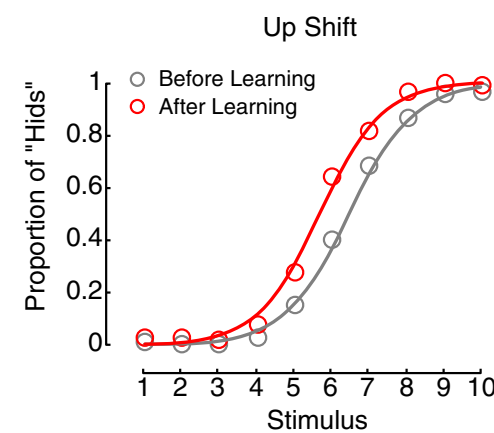

D

Head-to-Hid Continuum

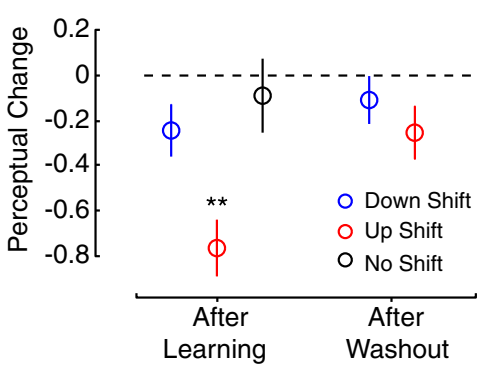

Figure 5. Learned changes in speech movements drive changes in speech perception. $\boldsymbol{A}-\boldsymbol{C}$, Show psychometric functions fit to the average proportion of hid responses (represented by the unfilled circles) at each stimulus before (gray lines) and after (colored speech motor learning. $\boldsymbol{D}$, After speech motor learning, a significant change in the perceptual boundary was observed in the 列 only observed when learned changes in production fell into the phonetic range of the perceptual test. The perceptual change disappeared after washout trials that eliminated motor learning; ${ }^{* *} p<0.01$.

\section{Head-to-Hid Continuum}

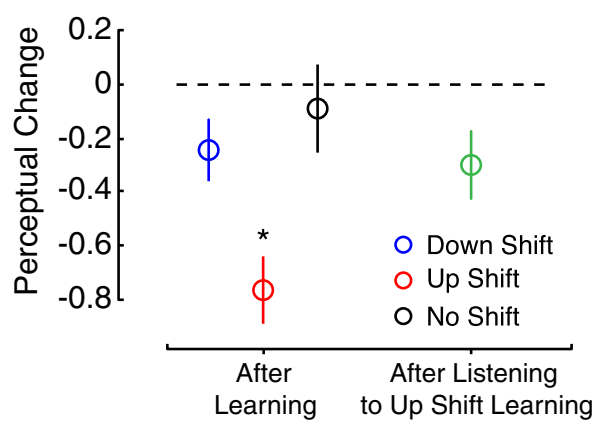

Figure 6. The effect of listening to speech motor learning on speech perception. Listening to speech motor learning in which learned changes in speech production fell into the phonetic range of the perceptual test caused a change in speech perception (green circle). However, this perceptual change was not as great as actually performing the same behavior (red circle), and it was no different from the perceptual change that occurred after repeatedly producing a word (black circle) or performing speech motor learning in which the perception of learning fell into the phonetic range of the perceptual test (blue circle).

the subsequent perceptual classification of fewer auditory stimuli as head. This is as if subjects were becoming more selective about the stimuli that constitute a satisfactory example of the training utterance. One possibility is that repetition of the utterance head results in perceptual suppression, an effect that has been referred to as selective adaptation or repetition suppression (Cole et al., 1975). The idea is that repeatedly hearing a word reduces perceptual sensitivity to that particular item and leads to an increase in the identification of other alternative stimuli in perceptual tests. 
While this could be a possible interpretation for the present results, there are a number of reasons for doubt. First, perceptual change in the present study is observed only in conjunction with motor learning. Changes to the perceptual classification of speech sounds are not observed under conditions where subjects repeat these same utterances while receiving normal speech feedback. Moreover, an explanation based on suppression would not, on its own, explain why the specific changes that are observed in the present study are related to speech movements associated with adaptation rather than with the auditory inputs during learning. The present results are thus able to situate the observed perceptual changes on the sensorimotor continuum, an aspect of the data that is not explored in earlier reports of this phenomenon.

The idea that there is suppression of sensory signals during movement has likewise been described in a number of reports focused on motor control rather than on speech production per se. The effects observed here may be related to these, so-called, efference copy or corollary discharge phenomena (Sperry, 1950; von Holst, 1954). However, the present effects also differ in notable ways. Evidence for efference copy is typically limited to transient sensory changes in conjunction with movement (Papakostopoulos et al., 1975; Rushton et al., 1981; Starr and Cohen, 1985; Bernier et al., 2009). The effects here persist well beyond the end of movement, throughout the perceptual measurement phase. Nevertheless the effects on perceptual systems of motor outflow during movement may well be the means by which these more durable effects arise. The fact that the persistence of perceptual change is limited to the period in which adaptation is maintained would be consistent with this interpretation.

It was found that measures of perceptual change were not correlated with the magnitude of adaptation. This is consistent with the findings of Shiller et al. (2009) and also with studies of perceptual recalibration in visuomotor adaptation (Cressman and Henriques, 2009). In contrast, correlations between speech motor adaptation and perceptual change have been observed with altered somatosensory feedback (Nasir and Ostry, 2009). In limb movement studies, also involving altered somatosensory feedback, perceptual change is likewise found to be correlated with the magnitude of learning (Ostry et al., 2010; Mattar et al., 2013). However, the presence of a correlation is unlikely to be due to experimental manipulations of somatosensory feedback alone. Indeed, the present experiments and those involving visuomotor rotation also involve altered somatosensory feedback in the sense that adaptation requires that subjects tolerate somatosensory error to correct for auditory or visual perturbations.

Shiller et al. (2009) report a result that seems inconsistent with the present finding. They observed changes in the perception of a /s- $\int /$ contrast after sensorimotor adaptation resulted in auditory inputs in the /s- $\int /$ perceptual range. However, there are differences between the two studies that are worth noting. The fact that fricatives and vowels rely on different kinds of spectral cues may provide a possible explanation for the diverging results. Another important difference is that sibilant fricatives ( $\mathrm{s}$ vs $\int$ ) have sharp perceptual boundaries compared with vowels, which in principle may make it easier to detect a small change in boundary location. It is also worth noting that Shiller et al. (2009) could not examine the effect of placing learned changes in production into the range of their perceptual test as there is no other phoneme contrast on the "side" of the production changes.

The effects of motor learning on perceptual systems have been reported most extensively in the context of human limb movement (Haith et al., 2008; Cressman and Henriques, 2009; Ostry et al., 2010). The findings are consistent with those observed here for speech motor learning in that perceptual boundary changes occur in the part of the workspace associated with the compensatory action. There is, in addition, evidence in both speech and limb movement that the effects of learning also flow in the opposite direction, that is, from sensory to motor networks such that perceptual learning changes sensory as well as motor function and motor areas of the brain. Shifts in perceptual acuity can occur when there is an uneven distribution of speech sounds to categorize (Clayards et al., 2008) or when listening to a foreign accent (Clarke and Garrett, 2004). In studies of perceptual learning in speech, brief periods of perceptual training result in persistent changes in the extent of speech motor learning (Lametti et al., 2014). Similarly, in limb movement, changes in perceptual acuity, as a result of perceptual training, result in improvements in both the rate and extent of subsequent motor learning (Darainy et al., 2013). In neuroimaging studies, somatosensory perceptual training results in changes in functional connectivity in frontal motor networks (Vahdat et al., 2014). The neuroimaging result in particular suggests that, in human limb movement, a portion of the change that occurs in brain motor areas during motor learning tasks may actually be the result of perceptual learning.

The present findings on perceptual change in speech motor learning thus need to be considered in the context of evidence that sensory input on its own can drive perceptual change and that perceptual learning alters motor learning and results in changes to motor areas of the brain. Reconciling the roles of perceptual and motor learning as determinants of human motor learning in general remains a significant challenge.

In the context of speech perception, the results suggest that brain areas that produce speech shape speech perception. This idea has previously been explored using noninvasive brain stimulation. In this work, transcranial magnetic stimulation is used to disrupt the neural functioning of speech motor areas and changes in speech perception are subsequently observed (Meister et al., 2007; D'Ausilio et al., 2009; Möttönen and Watkins, 2009, 2012 ). The implication of this work is that speech motor areas contribute to the perception of speech sounds, a provocative idea first proposed by the motor theory of speech perception (Liberman et al., 1967). The current findings provide additional evidence that speech motor areas contribute to speech perception. Adaptation to altered auditory feedback can be viewed as a behavioral alteration of the speech motor system. In this case, systematic changes in speech perception were observed when the motor system was in the adapted state. This supports the idea that motor areas play a role in speech perception.

In summary, we have shown that speech motor learning results in systematic changes to perceptual classification of speech sounds. By using speech adaptation as an experimental model, we have been able to separate changes to perceptual function that are attributable to sensory inputs during learning from those associated with the motor commands necessary to produce the adapted movements and sounds. In two separate studies we find that the perceptual change occurs in the part of the sound space that is associated with the compensatory movements required to generate the corrected speech sounds, and not in the part of the sound space associated with the auditory inputs that drive adaptation. The perceptual changes that accompany speech motor learning are thus substantially linked to the motor process of adaptation.

\section{References}

Bernier PM, Burle B, Vidal F, Hasbroucq T, Blouin J (2009) Direct evidence for cortical suppression of somatosensory afferents during visuomotor adaptation. Cereb Cortex 19:2106-2113. CrossRef Medline 
Cai S, Ghosh SS, Guenther FH, Perkell JS (2010) Adaptive auditory feedback control of the production of formant trajectories in the Mandarin triphthong /iau/ and its pattern of generalization. J Acoust Soc Am 128:20332048. CrossRef Medline

Clarke CM, Garrett MF (2004) Rapid adaptation to foreign-accented English. J Acoust Soc Am 116:3647-3658. CrossRef Medline

Clayards M, Tanenhaus MK, Aslin RN, Jacobs RA (2008) Perception of speech reflects optimal use of probabilistic speech cues. Cognition 108: 804-809. CrossRef Medline

Cole RA, Cooper WE, Singer J, Allard F (1975) Selective adaptation of English consonants using real speech. Percept Psychophys 18:227-244. CrossRef

Cressman EK, Henriques DY (2009) Sensory recalibration of hand position following visuomotor adaptation. J Neurophysiol 102:3505-3518. CrossRef Medline

Darainy M, Vahdat S, Ostry DJ (2013) Perceptual learning in sensorimotor adaptation. J Neurophysiol 110:2152-2162. CrossRef Medline

D’Ausilio A, Pulvermüller F, Salmas P, Bufalari I, Begliomini C, Fadiga L (2009) The motor somatotopy of speech perception. Curr Biol 19:381385. CrossRef Medline

Haith A, Jackson C, Miall C, Vijayakumar S (2008) Unifying the sensory and motor components of sensorimotor adaptation. La Jolla, CA: Advances In Neural Information Processing Systems.

Houde JF (2009) There's more to speech perception than meets the ear. Proc Natl Acad Sci U S A 106:20139-20140. CrossRef Medline

Houde JF, Jordan MI (1998) Sensorimotor adaptation in speech production. Science 279:1213-1216. CrossRef Medline

Lametti DR, Nasir SM, Ostry DJ (2012) Sensory preference in speech production revealed by simultaneous alteration of auditory and somatosensory feedback. J Neurosci 32:9351-9358. CrossRef Medline

Lametti DR, Krol SA, Shiller DM, Ostry DJ (2014) Brief periods of auditory perceptual training can determine the sensory targets of speech motor learning. Psychol Sci 25:1325-1336. CrossRef Medline

Liberman AM, Cooper FS, Shankweiler DP, Studdert-Kennedy M (1967) Perception of the speech code. Psychol Rev 74:431-461. CrossRef Medline

Mattar AA, Darainy M, Ostry DJ (2013) Motor learning and its sensory effects: time course of perceptual change and its presence with gradual introduction of load. J Neurophysiol 109:782-791. CrossRef Medline

Meister IG, Wilson SM, Deblieck C, Wu AD, Iacoboni M (2007) The essential role of premotor cortex in speech perception. Curr Biol 17:1692-1696. CrossRef Medline

Möttönen R, Watkins KE (2009) Motor representations of articulators contribute to categorical perception of speech sounds. J Neurosci 29:98199825. CrossRef Medline
Möttönen R, Watkins KE (2012) Using TMS to study the role of the articulatory motor system in speech perception. Aphasiology 26:1103-1118. CrossRef Medline

Nasir SM, Ostry DJ (2009) Auditory plasticity and speech motor learning. Proc Natl Acad Sci U S A 106:20470-20475. CrossRef Medline

Ostry DJ, Darainy M, Mattar AA, Wong J, Gribble PL (2010) Somatosensory plasticity and motor learning. J Neurosci 30:5384-5393. CrossRef Medline

Papakostopoulos D, Cooper R, Crow HJ (1975) Inhibition of cortical evoked potentials and sensation by self-initiated movement in man. Nature 258:321-324. CrossRef Medline

Purcell DW, Munhall KG (2006) Compensation following real-time manipulation of formants in isolated vowels. J Acoust Soc Am 119:2288 2297. CrossRef Medline

Rochet-Capellan A, Ostry DJ (2011) Simultaneous acquisition of multiple auditory-motor transformations in speech. J Neurosci 31:2657-2662. CrossRef Medline

Rushton DN, Rothwell JC, Craggs MD (1981) Gating of somatosensory evoked potentials during different kinds of movement in man. Brain 104:465-491. CrossRef Medline

Shiller DM, Sato M, Gracco VL, Baum SR (2009) Perceptual recalibration of speech sounds following speech motor learning. J Acoust Soc Am 125: 1103-1113. CrossRef Medline

Shiller DM, Gracco VL, Rvachew S (2010) Auditory-motor learning during speech production in 9-11-year-old children. PLoS One 5:e12975. CrossRef Medline

Sperry RW (1950) Neural basis of the spontaneous optokinetic response produced by visual inversion. J Comp Physiol Psychol 43:482-489. CrossRef Medline

Starr A, Cohen LG (1985) 'Gating' of somatosensory evoked potentials begins before the onset of voluntary movement in man. Brain Res 348:183186. CrossRef Medline

Vahdat S, Darainy M, Milner TE, Ostry DJ (2011) Functionally specific changes in resting-state sensorimotor networks after motor learning. J Neurosci 31:16907-16915. CrossRef Medline

Vahdat S, Darainy M, Ostry DJ (2014) Structure of plasticity in human sensory and motor networks due to perceptual learning. J Neurosci 34:24512463. CrossRef Medline

Villacorta VM, Perkell JS, Guenther FH (2007) Sensorimotor adaptation to feedback perturbations of vowel acoustics and its relation to perception. J Acoust Soc Am 122:2306-2319. CrossRef Medline

von Holst E (1954) Relations between the central nervous system and the peripheral organ. Br J Anim Behav 2:89-94. CrossRef 